\title{
A Influência dos Processos de Fundo sobre um Registro Fóssil de Cocolitoforídeos na Bacia de Pelotas
}

\author{
The influence of Bottom Processes on a Coccolithophore Fossil Record in Pelotas Basin
}

Juliana de Freitas Gonçalves $\odot$ \& Adriana Leonhardt

Universidade Federal do Rio Grande, Instituto de Oceanografia, Núcleo de Oceanografia Geológica, Laboratório de Paleoceanografia e Palinologia. Rio Grande, Rio Grande do Sul, Brasil

E-mails: goncalvesjuliana.f@gmail.com; adriana.leonhardt@yahoo.com.br

\section{Resumo}

\begin{abstract}
Baseado no registro de cocolitoforídeos do testemunho sedimentar SIS 188, recuperado do talude da Bacia de Pelotas, este trabalho buscou esclarecer a relação entre a deposição de carbonato e da matéria orgânica com a produtividade primária, assim como identificar os processos oceanográficos que a regula ao longo dos eventos climáticos marcantes do Quaternário tardio. O testemunho analisado contém os Estágios Isotópicos Marinhos 1, 2 e 3. As espécies Florisphaera profunda, habitante da zona fótica inferior, Emiliania huxleyi e Gephyrocapsa spp. dominam a associação ao longo de todo o intervalo. As ocorrências secundárias de Calcidiscus leptoporus e Helicosphaera spp. (as quais possuem afinidade com condições de ressurgência) e Umbilicosphaera spp. (que apresenta preferência por ambientes oligotróficos e coluna d'água estratificada) auxiliaram na interpretação da produtividade. Os cocolitoforídeos contribuíram para a transferência de carbonato e matéria orgânica para o fundo oceânico. Entretanto, especialmente durante o EIM 3, a deposição de carbono orgânico parece ser controlada principalmente pelas propriedades químicas das massas d'água de fundo. Durante o EIM 3, a associação de cocólitos também é influenciada pela velocidade da corrente de fundo, que os transporta juntamente com os sedimentos. As condições de fundo mudam durante o EIM 2, permitindo o acúmulo de cocólitos, $\mathrm{CaCO}_{3}$ e COT. No EIM 1, especialmente no Holoceno, todos os proxies apontam para um intervalo de maior produtividade.
\end{abstract}

Palavras-chave: Carbono orgânico; Carbonato; Atlântico Sudoeste

\section{Abstract}

Based in the coccolithophore record of sediment core SIS 188, recovered from Pelotas Basin slope, this study aims to elucidate the relation between carbonate and organic matter deposition with primary productivity, as well as identify the oceanographic processes which regulate it through the climate events of late Quaternary. The analyzed core covered the Marine Isotope Stages 1, 2 and 3. The species Florisphaera profunda, a lower photic zone dweller, Emiliania huxleyi and Gephyrocapsa spp. dominate the assemblage across the entire interval. The secondary occurrence of Calcidiscus leptoporus and Helicosphaera spp. (which have affinities with upwelling conditions) and Umbilicosphaera spp. (which prefers oligotrophic environments and stratified water column) were useful for productivity interpretation. Coccolithophores contributed to transferring carbonate and organic matter to the ocean bottom. However, mainly during MIS 3, deposition of organic carbon seems to be controlled mostly by chemical properties of deep-water masses. During MIS 3, the coccoliths assemblage is also influenced by bottom current velocity that transports them together with sediments. Bottom conditions changed during MIS 2, allowing the accumulation of coccoliths, $\mathrm{CaCO}_{3}$ and TOC. During MIS 1, specially the Holocene, all the proxies point to an interval of enhanced productivity.

Keywords: Organic carbon; Carbonate; Southwestern Atlantic 


\section{Introdução}

As glaciações e interglaciações do Quaternário são intervalos cada vez mais investigados com o objetivo de compreender o sistema climático atual. As principais mudanças climáticas registradas no Quaternário são controladas pelos parâmetros orbitais, embora as trocas de $\mathrm{CO}_{2}$ entre oceano e atmosfera também possuam um papel importante (Bigg, 2003).

A produtividade marinha cumpre um papel crucial na regulação do clima impactando diretamente o ciclo do carbono, seja através do consumo e fixação de $\mathrm{CO}_{2}$ pela atividade fotossintética do fitoplâncton, seja pela precipitação de carbonato mediada por organismos marinhos (Loubere et al., 2007). Cocolitoforídeos são algas cosmopolitas muito importantes neste contexto. Estas algas são caracterizadas por produzirem um exoesqueleto composto de diminutas placas calcárias, os cocólitos, que ao se sedimentarem no fundo oceânico podem ficar preservadas ao longo do tempo geológico (Billard \& Inouye, 2004). Assim, estudos de associações de cocolitoforídeos são consideradas excelentes ferramentas em análises paleoceanográficas. A abundância relativa da espécie Florisphaera profunda é um dos proxies mais conhecidos (Molfino \& Mcintyre, 1990; Flores et al., 2000).

As reconstituições de paleoprodutividade auxiliam a revelar potenciais conexões entre os processos oceanográficos regionais e mudanças globais (e.g. Pereira et al., 2018). O Atlântico Sul é uma região por onde fluem massas de água originadas nas altas latitudes do Atlântico Norte e Oceano Austral, contribuindo para a manutenção do equilíbrio climático. É também uma área em que águas ricas em nutrientes provenientes do Oceano Austral emergem (Anderson et al., 2009). Muitos trabalhos sobre a paleoprodutividade vem sendo desenvolvidos, principalmente considerando a margem leste da bacia oceânica (e.g. Mollenhauer et al., 2002; Hernández-Almeida et al., 2019) devido à magnitude do sistema de ressurgência de Benguela. No entanto, o registro sedimentar e fóssil pode apresentar um viés introduzido por processos que ocorreram durante ou após a deposição (e.g. Petró et al., 2016), afetando as interpretações paleoceanográficas.

Neste trabalho nós abordamos essa questão apresentando um registro paleoceanográfico com alta resolução temporal, abrangendo desde o EIM 3 até o EIM 1. A análise é baseada na variação da associação de cocólitos, dos proxies geoquímicos (como o conteúdo de carbonato e de carbono orgânico total nos sedimentos) e das características sedimentológicas em amostras coletadas em um testemunho localizado na Bacia de Pelotas.

\section{Características Oceanográficas Regionais}

O testemunho SIS 188 foi coletado no talude da Bacia de Pelotas, sul da Margem Continental Brasileira. A região de estudo é localizada ao norte da Frente Subtropical, o limite das águas quentes e salinas do giro subtropical em direção ao polo, originado na Zona de confluência BrasilMalvinas (Peterson \& Stramma, 1991).

A área é fortemente influenciada pela Corrente do Brasil (CB), originada do ramo meridional da Corrente Sul Equatorial, fechando a barreira oeste do Giro Subtropical (Peterson \& Stramma, 1991). A CB é responsável pelo transporte de calor e sal desde os trópicos para altas latitudes no Atlântico Sudoeste, transportando as águas quentes e salinas da Água Tropical (AT) e a Água Central do Atlântico Sul (ACAS). As camadas mais profundas da coluna d'água na região de estudo são compostas pela Água Profunda do Atlântico Norte (APAN) (formada na região Polar do Oceano Atlântico Norte), e pela Água de Fundo Antártica (AAF) (formada no Oceano Austral). A presença da AAF pode ser observada em profundidade maiores que $3000 \mathrm{~m}$ (Silveira et al., 2000).

Com relação a sedimentação, os principais fatores de controle na região do Atlântico Sul são a dissolução de carbonatos, o input terrestre e sua dispersão por correntes de turbidez, bem como o influxo, dissolução e deposição de material silicoso (Van Andel et al., 1977). A Bacia de Pelotas é também caracterizada por extensivas feições contorníticas que possuem disposição tanto erosiva como deposicional e também mistas (erosivas e deposicionais) (HernándezMolina et al., 2016), que são altamente relacionadas com interfácies de massas de água e depositadas ou retrabalhadas pela persistente ação das correntes de fundo, as quais podem ser modificadas localmente por canions submarinos e processo gravitavionais.

O nível da Profundidade de Compensação da Calcita (CCD - Carbonate compensation depth) varia de acordo com o fluxo da Água de Fundo Antártica em cada bacia do Oceano Atlântico Sul. Atualmente a CCD para nanofósseis calcários, grupo que inclui os cocolitoforídeos, está localizada entre $4050 \mathrm{~m}$ e $4500 \mathrm{~m}$ estando próxima ao limite superior da AAF no Canal de Vema (Melguen \& Thiede, 1974). O talude continental de onde os sedimentos foram recuperados está localizado milhares de metros acima da lisoclina do Atlântico Sul, consequentemente, a preservação dos cocólitos no testemunho é suficiente para obter seu registro e investigar sua contribuição para a bomba do carbono. 


\section{Material e Métodos}

\subsection{Testemunho Sedimentar Marinho}

O testemunho sedimentar SIS 188 foi recuperado do talude continental sul brasileiro $\left(-29.221286^{\circ}-47.283805^{\circ}\right)$ (Figura 1), sob $1514 \mathrm{~m}$ de profundidade. O testemunho consiste majoritariamente de lamas hemipelágicas levemente carbonáticas, com porções menores de lama levemente arenosa e camadas ricas em matéria orgânica (Figura 2). Foram analisados os $365 \mathrm{~cm}$ do testemunho. As amostras foram retiradas a cada $2 \mathrm{~cm}$ e peneiradas com malhas de $63 \mu \mathrm{m}$ e $125 \mu \mathrm{m}$. Todas as amostras foram secas em estufa a $50^{\circ} \mathrm{C}$ para confecção das lâminas para cocolitoforídeos.

\subsection{Modelo de Idade}

O modelo de idade (Figura 2) foi estabelecido correlacionando os registros de isótopos de oxigênio $\left(\delta^{18} \mathrm{O}\right)$ nas testas do foraminífero planctônico Globigerinoides ruber, que reflete a composição isotópica da água no topo da camada de mistura (Ravelo \& Fairbanks, 1992), com a curva LS16 para o Atlântico Sul de Lisiecki \& Stern (2016). As análises isotópicas foram realizadas no Laboratório de Espectometria de Massa da Universidade da California. Quatro datações por radiocarbono foram realizadas nas testas do foraminífero planctônico G. ruber no Laboratório de Radiocarbono da Universidade Federal Fluminense (LACUFF). As datações por radiocarbono foram corrigidas para o efeito reservatório (Delta $\mathrm{R}=54.0+/-42.0$ ) e calibradas de acordo com a curva Marine13 (Reimer et al., 2013), usando o Calib Radiocarbon Program (Stuiver \& Reimer, 1993). As datações foram utilizadas para controlar a cronologia entre ambas as curvas. Finalmente, o modelo de idade foi construído através da interpolação linear entre os pontos de controle, usando o software Analyseries 2.0 (Paillard et al., 1996).

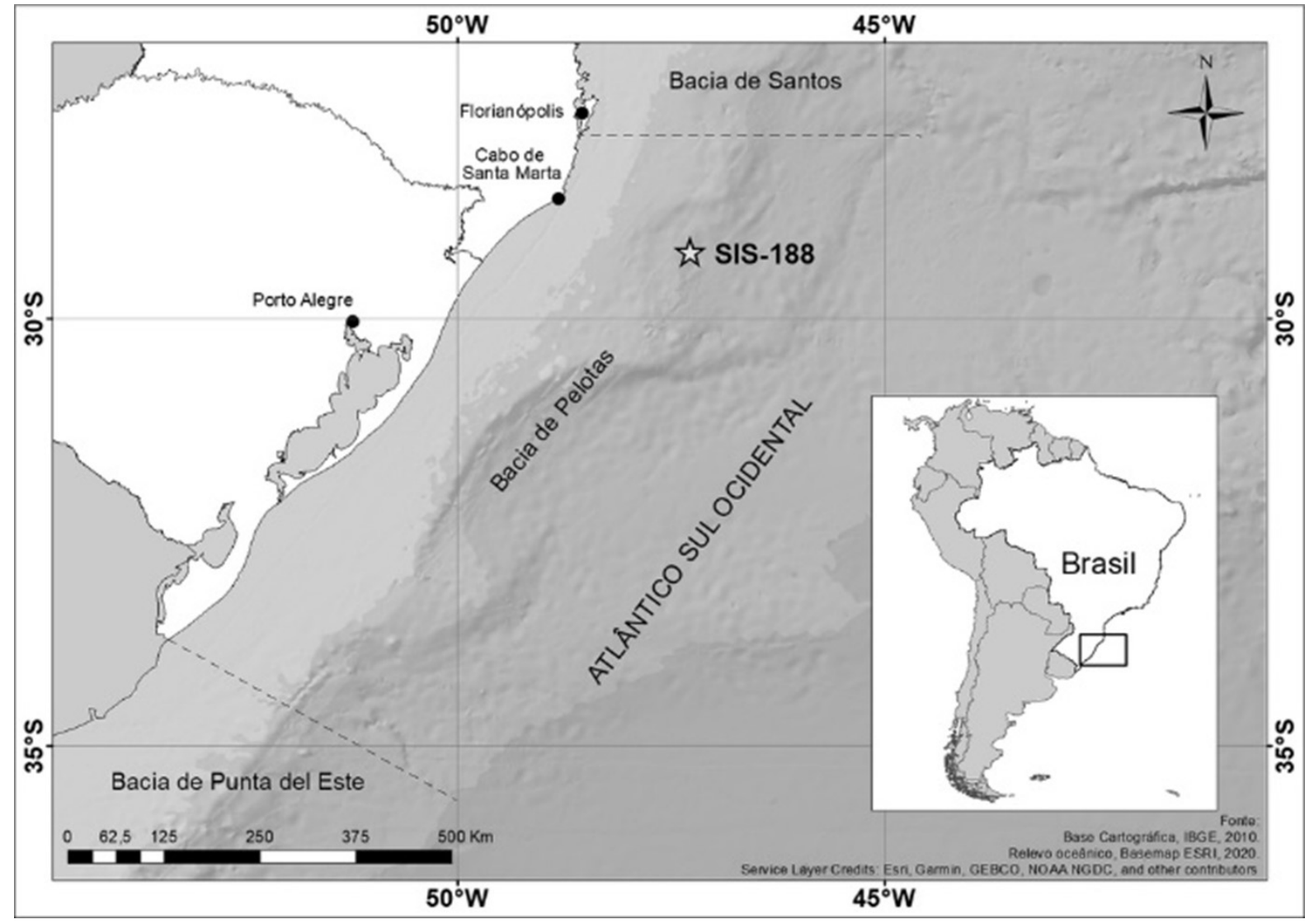

Figura 1 Localização do testemunho SIS 188 na Bacia de Pelotas.

Fonte da base cartográfica: IBGE (2010). 


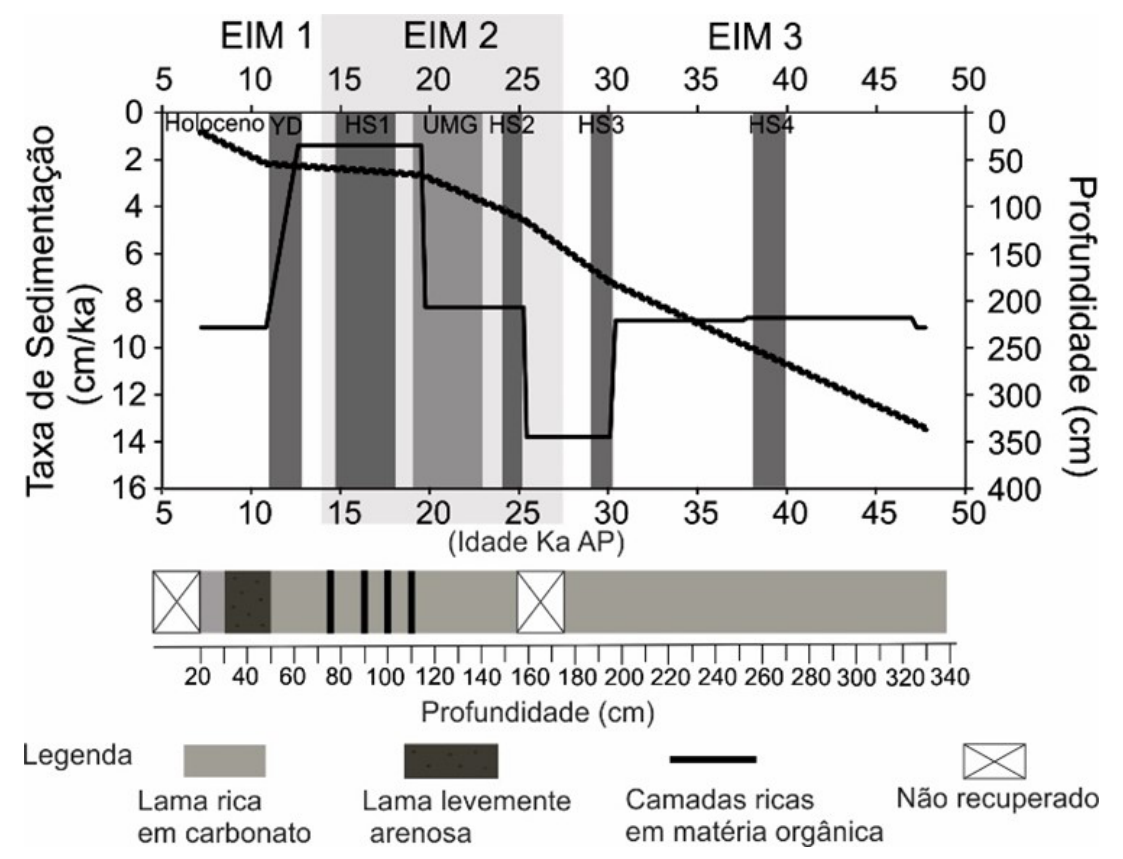

Figura 2 Modelo de idade-profundidade, taxas de sedimentação e a representação litológica do testemunho SIS 188. A linha pontilhada indica a variação da idade de acordo com a profundidade e a linha contínua indica a taxa de sedimentação. EIM= Estágio Isotópico Marinho, YD= Younger Dryas e HS= Heinrich Stadial.

\subsection{Medidas do Tamanho de Grão}

As medidas do tamanho de grão foram realizadas no Centro de Estudos Costeiros e Oceânicos (CECO) da Universidade Federal do Rio Grande do Sul, Brasil. Essas medições foram realizadas no intuito de identificar variações nas médias granulométricas na fração do sedimento compreendida entre $10 \mu \mathrm{m}$ e $63 \mu \mathrm{m}$. As análises foram obtidas utilizando o analisador de tamanho de partícula Horiba-Partica-LA-950 que se baseia no método de difração a laser. As partículas dispersam a luz em um ângulo determinado pelo seu tamanho. O padrão de dispersão da luz definido pela intensidade e ângulo será transformado em distribuição de tamanho de partícula como resultado (Horiba Instruments, 2016).

\subsection{Contagem de Cocólitos e Cálculos}

Com a finalidade de estimar a abundância absoluta de cocólitos por grama de sedimento seco, as amostras foram preparadas usando o método de dissolução e pipetagem descrito por (Koch \& Young, 2007). Após secagem em estufa das amostras, 0.1-0.2 $\mathrm{g}$ da fração fina $(<63 \mu \mathrm{m})$ foram diluídas em $10 \mathrm{ml}$ de água destilada, e posteriormente levadas ao ultrassom para melhor desagregação. As amostras foram diluídas mais uma vez em $5 \mathrm{ml}$ de água destilada e $500 \mu 1$ dessa segunda solução foram pipetadas em uma lamínula sobre uma placa aquecedora plana, para que pudesse secar rapidamente. As lamínulas foram montadas sobre a lâmina utilizando bálsamo do canadá. $\mathrm{O}$ uso de uma amostra com peso conhecido sobre uma área conhecida permite a contagem de cocólitos por campo visual ser convertida em números de espécimes por grama de sedimento.

O número de cocólitos por grama de sedimento foi calculado de acordo com Koch \& Young (2007):

Número de espécimes por grama de sedimento $=\frac{A * N}{f^{*} n * W}$

Onde A é a área da lamínula; fé a área de um campo visual; $\mathrm{n}$ é o número de campos visuais contados; $\mathrm{W}$ corresponde ao peso do sedimento sobre a lamínula; e $\mathrm{N}$ é o número de espécimes contados.

As contagens de cocólitos foram realizadas utilizando um microscópio de luz polarizada com magnificação de 1000x. Um mínimo de 500 cocólitos foram contados em um número de campos visuais conhecidos. A identificação das espécies de cocolitoforídeos foi baseada em Antunes (2007) e em Young et al. (2020).

A Razão N (Flores et al., 2000) foi utilizada para medir a paleoprodutividade e monitorar a profundidade da nutriclina. Consiste em uma razão entre a abundância relativa de espécies oportunistas, que se beneficiam de um 
aumento na disponibilidade de nutrientes na camada fótica (Gephyrocapsa spp. e Emiliania huxleyi), e Florisphaera profunda, habitante da zona eufótica inferior, que se beneficia de uma nutriclina profunda.

$$
\mathrm{RN}=\frac{\% \text { E.huxleyi }+\% \text { Gephyrocapsa spp. }}{\% \text { E.huxleyi }+\% \text { Gephyrocapsa spp.+\%F.profunda }}
$$

Foram realizadas observações sobre os diferentes estados de preservação dos cocólitos para cada amostra. Cinco categorias foram distinguidas utilizando uma escala similar à de Flores et al. (2003). Muito boa preservação (categoria 5) implica pouca ou nenhuma evidência de dissolução de carbonato, com as características morfológicas dos cocólitos bem preservadas; boa preservação (categoria 4) é definida por alto conteúdo de carbonato com pouca fragmentação e características diagnósticas preservadas; preservação moderada (categoria 3) é caracterizada por alguma dissolução, mas com quase todos os espécimes identificados a nível de espécie; preservação pobre (categoria 2) possui dissolução severa e fragmentação, com muitos espécimes não distinguidos; e amostras estéreis (categoria 1), com muito pouco ou nenhum conteúdo de carbonato.

\subsection{Medidas de Conteúdo de Carbonato de Cálcio e Carbono Orgânico Total}

$\mathrm{O}$ conteúdo de $\mathrm{CaCO}_{3}$ foi determinado através do método de perda de massa por digestão ácida (Molnia, 1974), na qual as amostras carbonatadas com pesos conhecidos foram atacadas por ácido clorídrico $(\mathrm{HCl})$ a uma concentração de $10 \%$. O conteúdo de carbonato é representado pela diferença no peso antes e depois de sua remoção pelo ataque ácido.

O carbono orgânico total (COT) foi medido através do método de oxidação catalítica por combustão em um analisador shimadzu para TOC-L Series SSM-5000 ${ }^{a}$. Cerca de $30 \mathrm{mg}$ de amostra de sedimento seco em estufa $\left(40^{\circ} \mathrm{C}\right)$ foram aquecidas a $900^{\circ} \mathrm{C}$ em um ambiente rico em oxigênio, alcançando combustão total e convertendo o carbono presente na amostra a dióxido de carbono $\left(\mathrm{CO}_{2}\right)$, o qual é detectado por um analizador infravermelho de gás.

A área calculada no pico da curva gerada pelo dióxido de carbono detectado é proporcional ao total da concentração de carbono na amostra, comparada a uma curva de calibração. Para avaliar apenas o carbono inorgânico, as amostras foram acidificadas e oxidadas a $200^{\circ} \mathrm{C}$. $\mathrm{O} \mathrm{CO}_{2}$ gerado nesse processo é o conteúdo de carbono inorgânico na amostra. A concentração de COT final é definida subtraindo a concentração do carbono inorgânico da concentração do carbono total. Essas análises foram realizadas no Laboratório de Análises Geoquímicas da Pontíficia Universidade do Rio Grande do Sul, Porto Alegre, Brasil.

\subsection{Correlação entre as Variáveis}

Correlações entre as variáveis estudadas foram calculadas. A significância das correlações foram avaliadas por autoreamostragem, com 10000 iterações, $\alpha=0,05$. As análises foram realizadas usando o software para análises estatísticas MULTIV (Pillar, 1997).

\section{Resultados}

\subsection{Modelo de Idade}

As idades de radiocarbono calibradas utilizadas para criar o modelo de idade do testemunho SIS 188 estão apresentadas na Tabela 1 . O testemunho investigado compreende o período de tempo de 47,7 a 7,2 cal ka AP. Durante o período glacial, a taxa de sedimentação mostra os maiores valores no intervalo estudado. Ela não apresentou variações representativas durante o EIM 3, porém demonstra um pico na transição para o EIM 2. Durante o Último Máximo Glacial (UMG), uma leve diminuição é notada, seguida por valores muito baixos durante a deglaciação (de 19,5 ka, no final do UMG, a 12,6 ka). Logo antes do início do Holoceno, a taxa de sedimentação aumenta uma vez mais, alcançando valores similares aos do UMG.

Tabela 1 Calibração das idades. $A P=$ antes do presente e ID $L A B=$ identificação do laboratório.

\begin{tabular}{|c|c|c|c|c|c|}
\hline $\begin{array}{l}\text { Profundidade } \\
\text { (cm) }\end{array}$ & Espécies & $\begin{array}{c}\text { Idade } \\
\left({ }^{14} \mathrm{C} \text { anos } \mathrm{AP}\right)\end{array}$ & Erro (anos) & $\begin{array}{l}\text { Idades calibradas } \\
\text { (cal anos AP) }\end{array}$ & ID Lab \\
\hline 21 & G. ruber & 6725 & \pm 31 & 7204 & LACUFF 170210 \\
\hline 54 & G. ruber & 9921 & \pm 34 & 10812 & LACUFF 170055 \\
\hline 113,5 & G. ruber & 21360 & \pm 59 & 25243 & LACUFF 170056 \\
\hline 180,8 & G. ruber & 26325 & \pm 77 & 30099 & LACUFF 170211 \\
\hline
\end{tabular}




\subsection{Análises Granulométricas}

As análises granulométricas foram realizadas com o intuito de identificar a variação da média granulométrica na fração entre 10 e $63 \mu \mathrm{m}$ (Figura 3), que caracteriza o tamanho do silte selecionável pela corrente. A média do tamanho dos grãos pertencentes à fração de 10 a 63 $\mu \mathrm{m}$ pode ser interpretada como um resultado da seleção controlada pela velocidade da corrente de fundo. As médias dos tamanhos de grão, excluindo-se o conteúdo carbonático, oscilaram entre $15,02 \mu \mathrm{m}$ e $37,75 \mu \mathrm{m}$. O tamanho médio do silte flutuou ao redor de $20 \mu \mathrm{m}$ entre 47,5 e $34 \mathrm{ka}$. Do final do EIM 3 ao início do EIM 2 (34-21,5 ka), a média foi menor, oscilando ao redor de $18 \mu \mathrm{m}$. A partir daí e durante o EIM 1, o tamanho médio aumentou, flutuando ao redor de $27 \mu \mathrm{m}$, e atingiu um pico de 37,75 $\mu \mathrm{m}$ há aproximadamente $8 \mathrm{ka}$.

Foi considerado apenas o sedimento não-carbonático porque as partículas biogênicas (como carapaças de foraminíferos) possuem tamanhos maiores que poderiam influenciar as medidas do material que é selecionado pela velocidade das correntes.

\subsection{Variações nas Associações de Cocolitoforídeos}

Amostras desprovidas de cocólitos e com os menores valores foram observadas principalmente durante o EIM 3. Os maiores números $\left(115 \times 10^{8}\right.$ cocólitos por grama de sedimento) foram registrados no EIM 1 e durante o Holoceno em $\sim 8.5 \mathrm{ka}$.

No total, 37 espécies e morfotipos foram identificadas, incluindo as espécies opostunistas Emiliania huxleyi
Lohmann (1902) Hay \& Mohler, in Hay et al., (1967), Gephyrocapsa spp. Kamptner (1943) (Gephyrocapsa “pequena", G. oceanica Kamptner (1943) e G. muellerae Bréhéret (1978), e Florisphaera profunda Okada \& Honjo (1973) (Figura 4), como também as espécies subordinadas Calcidiscus leptoporus Murray \& Blackman (1898) e Loeblich \& Tappan (1978), Helicosphaera spp. Kamptner (1954), Syracosphaera pulchra Lohmann (1902) e Umbilicosphaera spp. Lohmann (1902) (Figura 5).

F. profunda dominou a associação, alcançando valores maiores que $50 \%$ em algumas amostras durante a primeira metade do EIM 3. Sua porcentagem diminui no fim do EIM 3 (entre 32,7 e 27,9 ka), aumentando com o início do EIM 2 até o EIM 1. A segunda espécie mais abundante foi Emiliania huxleyi que mostrou suas maiores abundâncias relativas também durante o EIM 3. Essa abundância decai ligeiramente com o início do EIM 2, elevando-se novamente no EIM 1 (Figura 4).

Gephyrocapsa "pequena" obteve alta representatividade nas amostras, apresentando seus mais altos valores de abundancias relativas durante o EIM 3 e Holoceno. Gephyrocapsa oceanica $<5 \mu \mathrm{m}$ esteve presente em todo o testemunho, mas apresentou diminuição da porcentagem durante o EIM 2; após um aumento no início do EIM 1, suas porcentagens voltam a cair no Holoceno. A espécie Gephyrocapsa oceanica $>5 \mu \mathrm{m}$ revelou importantes valores de abundância relativa a partir do final do EIM 3, com quedas durante os eventos climáticos HS2, UMG, HS1 e Younger Dryas. Gephyrocapsa muellerae foi a espécie do gênero que apresentou a menor porcentagem ao longo do testemunho, apresentando um aumento expressivo no final do EIM 2 e início do EIM 1, contrapondo-se à tendência das demais espécies de Gephyrocapsa para este intervalo (Figura 4).

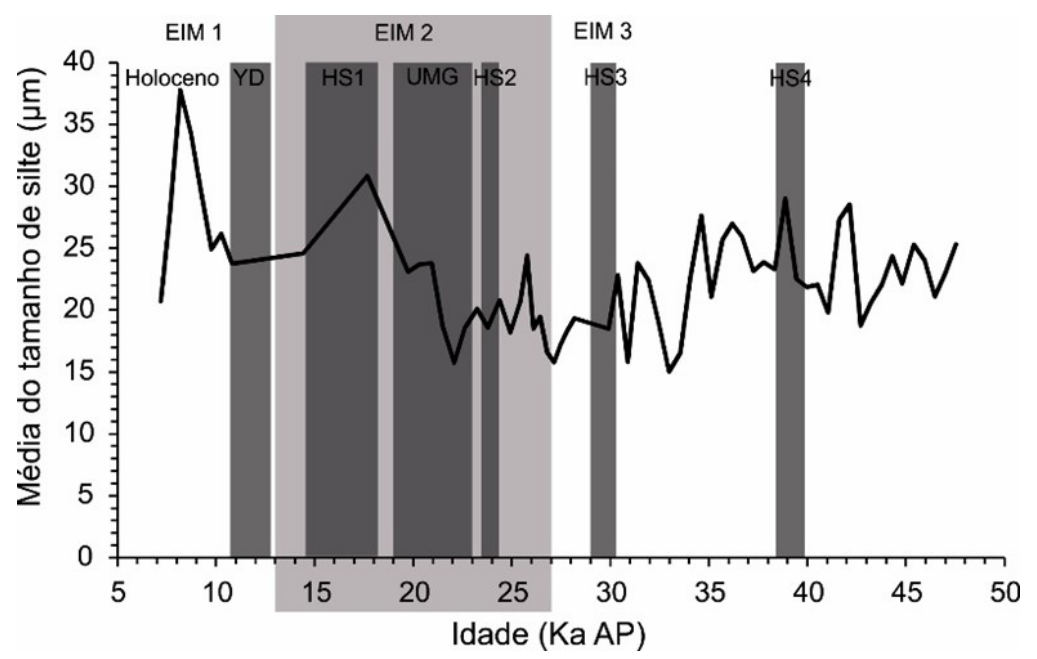

Figura 3 Média do tamanho de silte que pode ser selecionado pela corrente de fundo no testemunho SIS 188. EIM= Estágio Isotópico Marinho, HS= Heinrich Stadial, UMG= Último Máximo Glacial, YD= Younger Dryas e Ka (AP)= Mil anos antes do presente. 


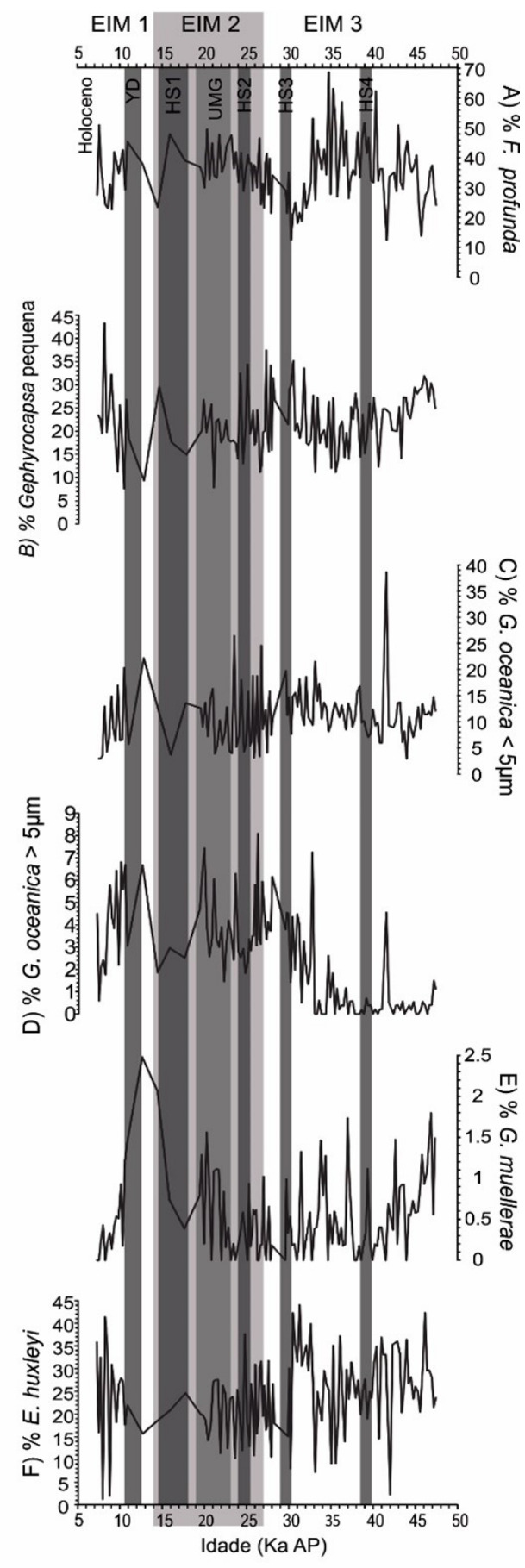

Figura 4 Comparação da oscilação das curvas de abundância relativa (AR) das espécies dominantes no testemunho SIS 188. A. Variação da AR de F. profunda; B. Variação da AR de Gephyrocapsa "pequena"; C. Variação da AR de G. oceanica morfotipo $<5 \mu \mathrm{m}$; D. Variação da AR de G. oceanica morfotipo $<5 \mu \mathrm{m}$; E. Variação da AR de G. mullerae; F. Variação da AR de E. huxleyi. EIM= Estágio Isotópico Marinho, HS= Heinrich Stadial, UMG= Último Máximo Glacial, YD= Younger Dryas e Ka (AP)= Mil anos antes do presente.

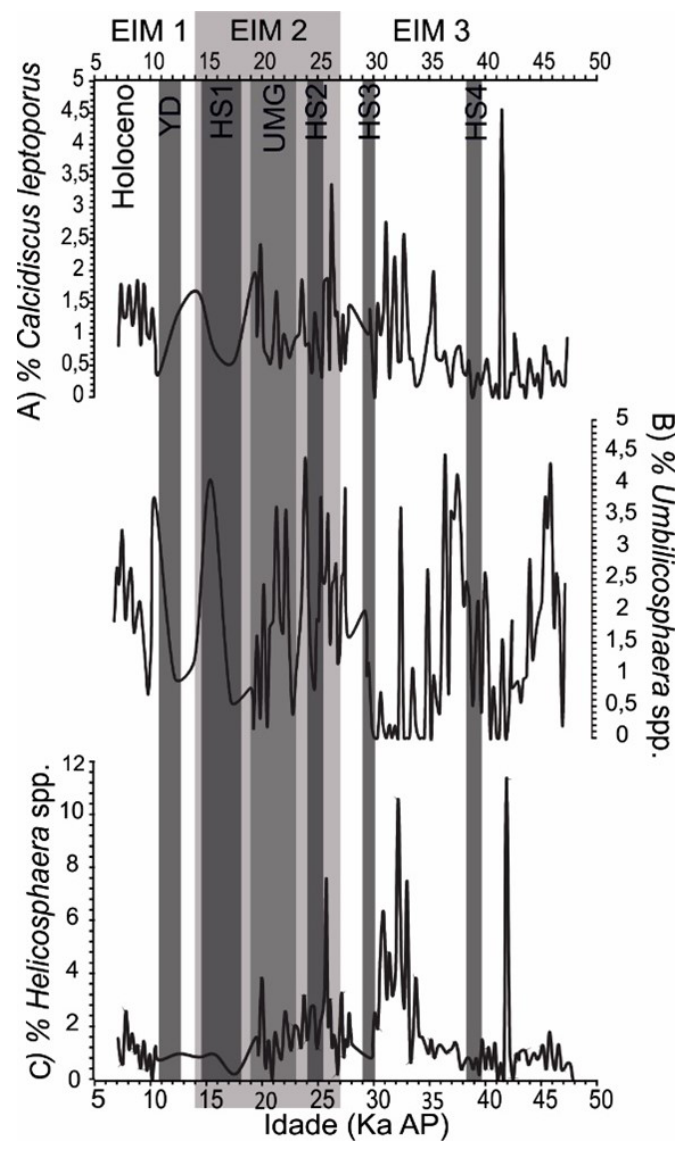

Figura 5 Comparação da variação entre as curvas de abundância relativa (AR) de espécies subordinadas que apresentam preferência pela coluna d'água estratificada $(B)$ e espécies indicadoras de condições de ressurgência $(A$ e $C$ ). A. Curva da variação da AR de Calcidiscus leptoporus; $B$. Curva da variação da AR de Umbilicosphaera spp.; C. Curva da variação da AR de Helicosphaera spp. EIM= Estágio Isotópico Marinho, HS= Heinrich Stadial, UMG= Último Máximo Glacial, YD= Younger Dryas e Ka (AP) $=$ Mil anos antes do presente.

A maioria das espécies identificadas não alcançou $1 \%$ do total da associação. Entretanto, algumas espécies subordinadas foram muito representativas na associação alcançando valores maiores que $10 \%$ em algumas amostras. A presença de C. leptoporus pode ser notada através dos três Estágios Isotópicos Marinhos, mas demonstra maior porcentagem desde o final do EIM $3(\sim 35,7 \mathrm{ka})$ até o Holoceno, quando está presente em todas as amostras. O percentual de espécies de Helicosphaera aumenta no final do EIM 3, de $\sim 32 \mathrm{ka} \mathrm{a} \sim 25 \mathrm{ka}$, mostrando um claro padrão de diminuição durante a deglaciação e Holoceno. O grupo Helicosphaera foi representado principalmente pela espécie $H$. carteri. Umbilicosphaera spp., representada principalmente por $U$. sibogae, demonstrou grande oscilação 
através do tempo, sendo notada frequentemente durante o Holoceno, quando representa mais de $1 \%$ da associação em quase todas as amostras (Figura 5).

\subsection{Medidas de Paleoprodutividade, Análises Geoquímicas e suas Correlações}

A curva da razão $\mathrm{N}$ indica alguns intervalos de maior paleoprodutividade, como ao redor de 47,5 - 41,7 ka, 33,3 - 29,9 ka e 10,4 - 7,9 ka e menores valores durante o EIM 2. Diferentemente, a curva de abundância absoluta de cocólitos (cocólitos por grama de sedimento) apresenta uma nítida tendência de elevação do final do EIM $3 \mathrm{em}$ direção ao Holoceno. A mesma tendência é demonstrada nas curvas de carbono orgânico total (COT) e de conteúdo de carbonato $\left(\mathrm{CaCO}_{3}\right)$ nos sedimentos.

As estimativas da preservação dos cocólitos nas amostras apresentaram preservação de moderada a boa, em geral. As amostras com preservação muito boa apareceram apenas durante o EIM 3 e o EIM 1.

A maior correlação obtida entre as variáveis ocorreu entre "número de cocólitos" e "conteúdo de carbonato de cálcio", de 0,7 . As variáveis "número de cocólitos" e "conteúdo de carbonato de cálcio" demonstraram boa correlação com "conteúdo de COT". "Tamanho médio do silte" apresentou correlação negativa com "número de cocólitos". As correlações de "tamanho médio do silte" com "conteúdo de carbonato" e com "conteúdo de COT" não foram consideradas significativas (Tabela 2).

Tabela 2 Correlação entre conteúdo de $\mathrm{CaCO}_{3}$ e número de cocólitos por grama de sedimento, correlações entre conteúdo de COT e número de cocólitos por grama de sedimento, Razão $\mathrm{N}$ e conteúdo de $\mathrm{CaCO}_{3}$, e correlações entre tamanho médio do silte e número de cocólitos, conteúdo de $\mathrm{CaCO} 3$ e conteúdo de COT. COT= carbono orgânico total e $\mathrm{CaCO}_{3}=$ carbonato de cálcio.

\begin{tabular}{|c|c|c|c|c|}
\hline & $\mathrm{CaCO}_{3}$ & СOT & Tamanho médio do silte & \\
\hline \multirow{2}{*}{$N^{\circ}$ de cocólitos } & 0,69748 & 0,49337 & $-0,34679$ & Correlação \\
\hline & 0,0001 & 0,0002 & 0,009 & p-valor \\
\hline \multirow{2}{*}{ Razão N } & & $-0,27407$ & & Correlação \\
\hline & & 0,0441 & & p-valor \\
\hline \multirow{2}{*}{$\mathrm{CaCO}_{3}$} & & 0,66267 & $-0,26144$ & Correlação \\
\hline & & 0,0001 & 0,055 & p-valor \\
\hline \multirow{2}{*}{ СOT } & & & -0.35178 & Correlação \\
\hline & & & 0.0557 & p-valor \\
\hline
\end{tabular}

\section{Discussão}

De acordo com nossos resultados, o EIM 3 apresenta o menor conteúdo de $\mathrm{CaCO}_{3}$, que aumenta progressivamente em direção ao EIM 2; a mesma tendência é verificada para a curva da abundância absoluta de cocólitos (Figura 6). Além da congruência nas tendências das curvas, a alta correlação encontrada entre essas variáveis $(0,7)$ (Tabela 2$)$ indica que há contribuição destes organismos no aporte de carbonatos para o fundo marinho. $\mathrm{O}$ mesmo já havia sido notado na área de estudo por Gonçalves \& Leonhardt (2016), principalmente durante o UMG. Além disso, a boa correlação $(0,66)$ entre $\mathrm{CaCO}_{3}$ e COT demonstra a influência dos cocolitoforídeos também no aporte de matéria orgânica para o fundo marinho.

No entanto, as curvas de COT e de cocólitos por grama apresentam uma tendência oposta à da Razão N (que monitora a profundidade da nutriclina) no EIM 3 (Figura 6). COT e Razão N apresentam correlação negativa (Tabela 2), indicando que a acumulação de COT não se relaciona à oscilação da produtividade primária conforme descrita por este proxy para este intervalo. Apesar disso, a porcentagem de outras espécies corrobora a interpretação para a paleoprodutividade baseada na Razão N. A abundância relativa de $F$. profunda é considerada um indicativo de estratificação das águas, já que essa espécie está adaptada a zona eufótica inferior. Umbilicosphaera spp. apresenta preferência por águas quentes e oligotróficas no Atlântico Sul (Baumann et al., 2016), apresentando um padrão de variação muito parecido ao de $F$. profunda. Já a ocorrência de Helicosphaera spp. está associada a maior disponibilidade de nutrientes e maior mistura da camada superficial da coluna d'água (Boeckel \& Baumann, 2006), enquanto Calcidiscus leptoporus apresenta maiores porcentagens em regiões de ressurgência, ricas em nutrientes (Baumann et $a l ., 2016)$. No testemunho SIS 188, as porcentagens de $C$. leptoporus acompanham as porcentagens de Helicosphaera spp., e ambas se contrapõem parcialmente às porcentagens de F. profunda. 


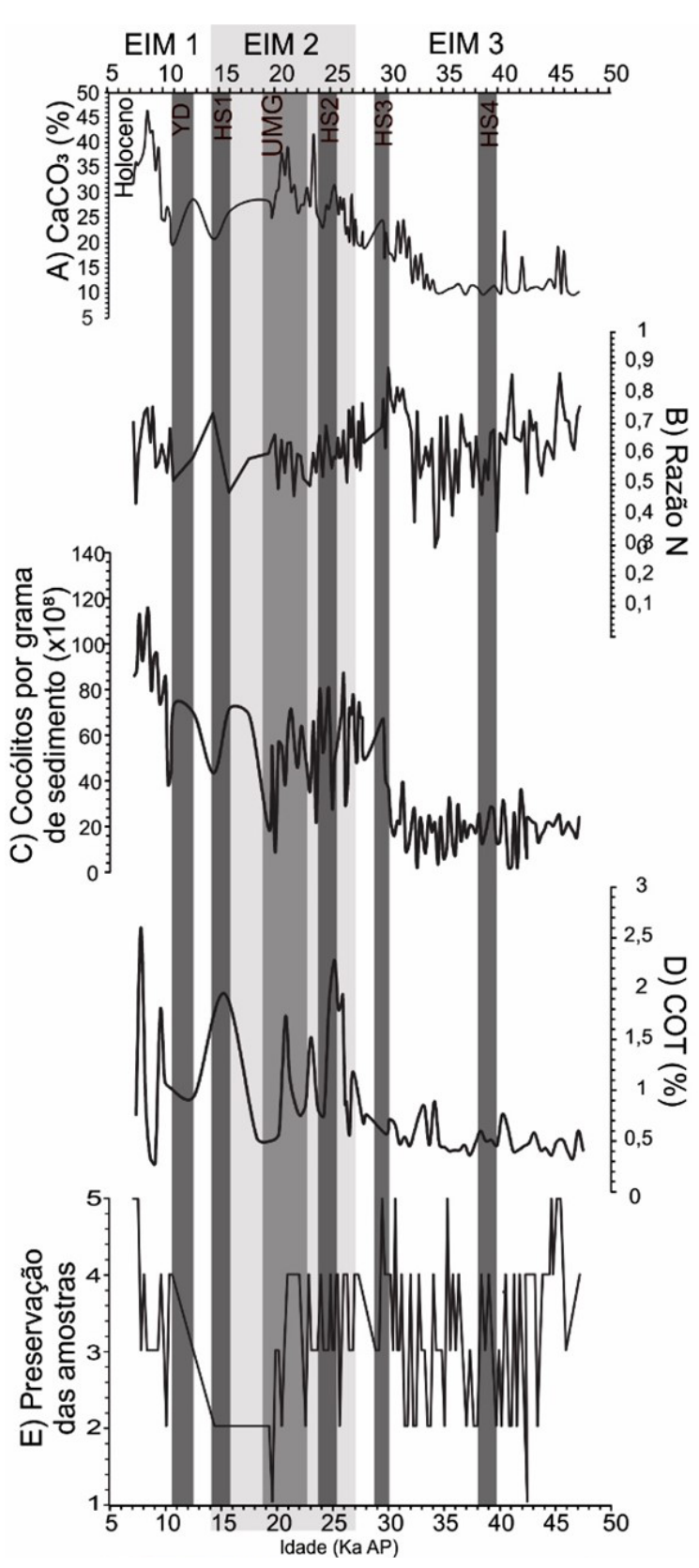

Figura 6 Comparação entre as curvas de proxies. A. Variação da curva do conteúdo de carbonato; $B$. Variação da curva da razão $\mathrm{N}$; C. Variação da curva de cocólitos por grama de sedimento; D. Variação da curva de carbono orgânico total; E. Variação da curva de preservação das amostras. EIM= Estágio Isotópico Marinho, HS= Heinrich Stadial, UMG= Último Máximo Glacial, $\mathrm{YD}=$ Younger Dryas e Ka (AP)= Mil anos antes do presente.

A princípio, o registro fossilífero de cocolitoforídeos documenta os processos em superfície associados à produtividade que afetam diretamente as suas associações. Entretanto, devido às particularidades do local onde o testemunho foi recuperado, processos que podem ocorrer durante ou após a deposição também devem ser considerados nas interpretações. Sabe-se que a taxa de sedimentação pode ser um dos fatores que controla a preservação da matéria orgânica já que determina, em parte, o tempo de exposição desta ao oxigênio. No entanto, durante o EIM 3 a taxa de sedimentação no local de estudo esteve bastante alta, alcançando valores de até $10 \mathrm{~cm} / \mathrm{ka}$ (Figura 2), enquanto o COT demonstra os menores valores no testemunho, o que indica que outros fatores são preponderantes sobre sua deposição ou preservação nesse período.

Uma outra hipótese para explicar o comportamento antagônico destes proxies no EIM 3 envolve as propriedades químicas das águas de fundo. Mollenhauer et al. (2004) sugerem que a sedimentação de COT está mais proximamente relacionada às propriedades químicas das massas de água e ao input de matéria orgânica terrígena para áreas profundas durante os intervalos glaciais do que, necessariamente, ao aumento da produtividade primária. De acordo com estes autores, a acumulação de carbono orgânico foi maior durante o UMG do que no Holoceno ao longo das margens continentais da África e da América do Sul e na região equatorial. Essa diferença foi associada, principalmente, à geometria das massas de água: a atuação preponderante de massas de água caracterizadas por maiores níveis de oxigênio promoveria maior degradação da matéria orgânica.

Curry \& Oppo (2005) usaram a composição isotópica de ${ }^{13} \mathrm{C}$ como traçador de massas de água no Atlântico Sul durante o último período glacial e verificaram que a massa de água centrada a $1500 \mathrm{~m}$ em $27^{\circ} \mathrm{S}$ representa a penetração mais ao sul da componente Norte (Água Intermediária do Atlântico Norte Glacial), que possui menores níveis de oxigênio do que a Água Intermediária Antártica moderna. Bohm et al. (2015), utilizando a razão ${ }^{231} \mathrm{~Pa} /{ }^{230} \mathrm{Th}$ como traçadora de massas de água, concluíram que a intensidade da Circulação de Revolvimento Meridional do Atlântico (AMOC - Atlantic meridional overturning circulation) durante o EIM 3 (um intervalo glacial mais ameno que o EIM 2), apesar de sofrer variações, estava fortalecida de forma similar à circulação do EIM 1. Assim, durante o EIM 3, uma AMOC vigorosa aumentaria a influência da Água Intermediária Antártica no local de estudo, degradando parte da matéria orgânica depositada no período.

Os baixos valores de cocólitos por grama de sedimento acompanhados de alta Razão N no EIM 3 poderiam ser explicados por uma dissolução de carbonatos aumentada neste intervalo. Porém, embora este intervalo seja acompanhado de baixa porcentagem de $\mathrm{CaCO}_{3}$ nos sedimentos, a dissolução não parece ser significativa porque as amostras sempre apresentaram uma preservação dos cocólitos de moderada a muito boa (Figura 6). 
A sedimentação atual na margem continental sul do Brasil é dominada pela dinâmica das massas de água oceânicas que determinam a redistribuição de sedimentos sobre a plataforma e na margem continental (Mahiques et al., 2011). Extensas áreas com características contorníticas ocorrem ao longo de toda a margem continental que, somadas às variações eustáticas e climáticas do Quaternário, controlam o padrão de empilhamento sedimentar (Hernández-Molina et al., 2016). O testemunho SIS 188 foi recuperado sob uma profundidade de $1514 \mathrm{~m}$ no talude continental, sobre o Terraço do Rio Grande, um depósito contornítico que está associado à competência do transporte do sedimento pelas correntes de fundo. Ao norte, na Bacia de Santos, Mahiques et al. (2011) associaram as menores taxas de sedimentação na plataforma externa e no talude superior ao maior fluxo da Corrente do Brasil, já que uma maior velocidade da corrente dificultaria a sedimentação. Assim, outra hipótese para explicar o registro do EIM 3 está relacionada à velocidade do fluxo da corrente de fundo do local de estudo.

A Corrente do Brasil flui na direção sudeste nos primeiros $500 \mathrm{~m}$ da coluna de água ao longo da margem continental sul brasileira e talude superior. Em profundidades intermediárias (entre 500 e $1800 \mathrm{~m}$, onde se encontra o testemunho SIS 188), as correntes do ramo oeste do giro subtropical se aproximam do Alto de Florianópolis, que delimita as Bacias de Pelotas e Santos, a cerca de 27$28^{\circ} \mathrm{S}$. Nesse ponto a $\mathrm{CB}$ se divide em dois ramos e forma a bifurcação de Santos: o ramo norte flui em direção nordeste, enquanto o ramo sul da bifurcação flui para sudoeste, ao longo do talude da Bacia de Pelotas, formando a Corrente de Contorno Oeste Intermediária (CCOI), que recircula a Água Intermediária Antártica (AIA) (Schmid et al., 2000).

O tamanho médio do silte encontrado no testemunho SIS 188 é baixo ao longo do EIM 3 (Figura 3), indicando menor velocidade de fluxo da corrente de fundo quando comparada a segunda metade do EIM 2 e ao EIM 1, e também permitindo uma maior deposição de sedimentos. Portanto, a baixa quantidade de cocólitos registrada para o EIM 3, acompanhada de um alta Razão N, pode deverse ao efeito da diluição dos cocólitos nos sedimentos associada a menor velocidade de fluxo da CCOI, que permitiu uma maior taxa de sedimentação. A correlação negativa encontrada entre estas duas variáveis (tamanho médio do silte e número de cocólitos) dá suporte a essa interpretação. No entanto, esta correlação não é muito alta $(-0,3)$, mostrando que outros fatores, como mudanças no tamanho da população de cocolitoforídeos, também interferem na flutuação dessa variável ao longo do tempo.

A relação entre os proxies muda ao longo do registro. No EIM 2, a taxa de sedimentação se mantém alta, enquanto as quantidades de cocólitos, $\mathrm{COT}$ e $\mathrm{CaCO}_{3}$ nos sedimentos aumentam consideravelmente, mostrando que as condições de fundo mudaram em relação ao EIM 3. No Holoceno, os proxies apontam para um intervalo de alta produtividade primária em superfície (maiores quantidades de cocólitos, COT e Razão N). Sabe-se que neste intervalo a AMOC estava totalmente reestabelecida (Adkins, 2013) com influência da Água Intermediária Antártica no fundo. A produtividade primária neste intervalo estava aumentada a ponto de permitir a boa preservação do carbono orgânico mesmo com a maior oxigenação do fundo em comparação ao EIM 2. Da mesma forma, o crescimento das populações de cocolitoforídeos foi tal que sua concentração nos sedimentos é a maior do registro, mesmo com o aumento da velocidade do fluxo da CCOI no fundo (indicada pelo aumento do tamanho médio do silte).

\section{Conclusão}

O testemunho SIS 188, retirado do talude da Bacia de Pelotas, foi estudado a partir da associação de cocolitoforídeos, dados geoquímicos e sedimentológicos. Os cocolitoforídeos foram contribuintes tanto de carbonato como de carbono orgânico para o fundo marinho no intervalo estudado (47,7 a 7,2 cal ka AP). No entanto, processos ocorridos durante ou após a deposição dos sedimentos e destes microfósseis alteraram seu registro. Durante o EIM 3, a baixa concentração de COT pode estar relacionada à exposição a águas de fundo com maior conteúdo de oxigênio, enquanto a baixa quantidade de cocólitos foi controlada pela alta taxa de sedimentação e a menor velocidade da corrente. Ao contrário, durante o Holoceno a produtividade aumentou o suficiente para permitir a preservação de altas quantidades de COT e de cocólitos por grama de sedimento seco, ainda que as águas de fundo estivessem oxigenadas e a velocidade da corrente fosse maior.

\section{Agradecimentos}

As autoras agradecem à CAPES (Coordenação de Aperfeiçoamento de Pessoal de Nível Superior) pelo suporte financeiro e bolsa de doutorado que possibilitaram a execução deste trabalho (CAPES processo n ${ }^{\circ} 88887.091729 / 2014-01$ ). A primeira autora também é grata ao PPG em Oceanologia da FURG pela oportunidade.

\section{Referências}

Adkins, J.F. 2013. The role of deep ocean circulation in setting glacial climates. Paleoceanography, 28:539-561. doi: 10.1002/palo.20046.

Anderson, R.F.; Ali, S.; Bradtmiller, L.I.; Nielsen, S.H.H.; Fleisher, M.Q.; Anderson, B.E. \& Burckle, L.H. 2009. Wind-Driven 
upwelling in theRise in Atmospheric CO2. Science, 323:14431448. doi: $10.1126 /$ science. 1167441 .

Antunes, R.L. 2007. Nanofósseis Calcários do Quaternario da Margem Continental Brasileira. Série Ciência Técnica Petróleo, 21: 76p.

Baumann, K.H.; Saavedra-Pellitero; M.; Böckel, B. \& Ott, C. 2016. Morphométrie, biogéographie et écologie des genres Calcidiscus et Umbilicosphaera dans l'Atlantique Sud. Revue de Micropaleontologie, 59: 239-251. doi: 10.1016/j. revmic.2016.03.001.

Bigg, G.R. 2003. The oceans and climate. $2^{\mathrm{a}}$ ed. Cambridge University Press, New York, 273p.

Billard, C. \& Inouye, I. 2004. What is nem in coccolithophore biology? In: Thierstein, H.R. \& Young, J.R. (Eds.). Coccolithophores. From molecular processes to global impact. Berlim, Springer, p.1-29.

Boeckel, B. \& Baumann, K. 2006. Coccolith distribution patterns in South Atlantic and Southern Ocean surface sediments in relation to environmental gradients, Deep-Sea Research I, 53:1073-1099. doi: 10.1016/j.dsr.2005.11.006.

Bohm, E.; Lippold, J.; Gutjahr, M.; Frank, M.; Blaser, P.; Antz, B., Fohlmeister J.; Frank, N.; Andersen, M.B. \& Deininger, M. 2015. Strong and deep Atlantic meridional overturning circulation during the last glacial cycle. Nature, 517:73-76. doi: 10.1038/nature14059.

Bréhéret, J.G. 1978. Formes nouvelles quaternaries et actualles de la famille des Gephyrocapsaceae (Cocolithophorides). Comptes Rendus Hebdomadaires des Séances de l'Académie des Sciences, Paris, Série D - Sciences Naturelles, 287: 447449.

Curry, W.B. \& Oppo, D.W. 2005. Glacial water mass geometry and the distribution of $\delta^{13} \mathrm{C}$ of $\Sigma \mathrm{CO}_{2}$ in the western Atlantic Ocean. Paleoceanography, 20:1-13. doi: 10.1029/2004PA001021.

Flores, J.A.; Bárcena, M.A. \& Sierro, F.J. 2000. Ocean-surface and wind dynamics in the Atlantic Ocean off Northwest Africa during the last 140000 years. Palaeogeography, Palaeoclimatology, Palaeoecology, 161:459-478. doi: 10.1016/S0031-0182(00)00099-7.

Flores, J.A.; Marino, M.; Sierro, F.J.; Hodell, D.A. \& Charles, C.D. 2003. Calcareous plankton dissolution pattern and coccolithophore assemblages during the last $600 \mathrm{kyr}$ at ODP Site 1089 (Cape Basin, South Atlantic): Paleoceanographic implications. Palaeogeography, Palaeoclimatology, Palaeoecology, 196:409-426. doi: 10.1016/S00310182(03)00467-X.

Gonçalves, J.F. \& Leonhardt, A. 2016. Contribuição dos cocolitoforídeos para o aporte de carbonato de cálcio durante o Último Máximo Glacial na Margem Continental Sul Brasileira. Quaternary and Environmental Geosciences, 07(1-2):1-5. doi: 10.5380/abequa.v7i1-2.45705.

Hay, W.W; Mohler, H.P; Roth, P.H.; Schmidt, R.R. \& Boudreaux, J.E. 1967. Calcareous nannoplankton zonation of the Cenozoic of the Gulf Coast and Caribbean-Antillean area, and transoceanic correlation. Transactions of the Gulf-Coast Association of Geological Societies, 17: 428-480.

Hernández-Almeida, I.; Ausín, B.; Saavedra-Pellitero M.; Baumann, K.H. \& Stoll, H.M. 2019. Quantitative reconstruction of primary productivity in low latitudes during the last glacial maximum and the mid-to-late Holocene from a global Florisphaera profunda calibration dataset. Quaternary Science Reviews, 205:166-181. doi: 10.1016/j.quascirev.2018.12.016.

Hernández-Molina, F.J.; Soto, M.; Piola, A.R.; Tomasini, J.; Preu, B.; Thompson, P.; Badalini, G.; Creaser, A.; Violante, R.A.; Morales, E.; Paterlini, M. \& De Santa Ana, H. 2016. A contourite depositional system along the Uruguayan continental margin: Sedimentary, oceanographic and paleoceanographic implications. Marine Geology, 378: 333-349. doi: 10.1016/j. margeo.2015.10.008.

Horiba Instruments. 2016. A guidebook to particle size analysis. Horiba Scientific, $31 \mathrm{p}$.

IBGE. 2010. Instituto Brasileiro de Geografia e Estatística. Disponível em <https://mapas.ibge.gov.br/bases-ereferenciais/bases-cartograficas/malhas-digitais $>$. Acesso em 20 jul. 2020 e 25 ago. 2020.

Kamptner, E. 1954. Untersuchungen über den Feinbau der Coccolithen. Archiv für Protistenkunde, 100: 1-90.

Koch, C. \& Young, J.R. 2007. A simple weighing and dilution technique for determining absolute abundances of coccoliths from sediment samples. Journal of Nannoplankton Research, 29: 67-69.

Lisiecki, L.E. \& Stern, J.V. 2016. Glacial Cycle. Paleoceanography, 31:1368-1394. doi: 10.1002/2016PA003002.

Loeblich, A.R. \& Tappan, H. 1978. The coccolithophorid genus Calcidiscus Kamptner and its synonyms. Journal of Paleontology, 52: 1390-1392.

Lohmann, H. 1902. Die Coccolithophoridae, eine Monographie der Coccolithen bildenden Flagellaten, zugleich ein Beitrag zur Kenntnis des Mittelmeerauftriebs. Archiv für Protistenkunde, 1: 89-165.

Loubere, P; Sidlecki, S.A. \& Bradtmiller, L.I. 2007. Organic carbon and carbonate fluxes: link to climate change. Deep Sea Research Part II: Topical Studies in Oceanography, 54 : 437-446.

Mahiques, M.M.; Sousa, S.H.M.; Burone, L.; Nagai, R.H.; Silveira, I.C.A.; Figueira, R.C.L.; Soutelino, R.G.; Ponsoni, L. \& Klein, D.A. 2011. Radiocarbon geochronology of the sediments of the São Paulo Bight (southern Brazilian upper margin). Anais da Academia Brasileira de Ciências, 83:817-834. doi: 10.1590/S0001-37652011005000028.

Melguen, M. \& Thiede, J. 1974. Facies distribution and dissolution depths of surface sediment components from the Vema Channel and the Rio Grande Rise (Southwest Atlantic Ocean). Marine Geology, 17: 341-353.

Molfino, B. \& Mcintyre, A. 1990. Precessional forcing of nutricline dynamics in the equatorial Atlantic. Science, 249:766-769. doi: 10.1126/science.249.4970.766.

Mollenhauer, G.; Schneider, R.R.; Jennerjahn, T.; Müller, P.J. \& Wefer, G. 2004. Organic carbon accumulation in the South Atlantic Ocean: Its modern, mid-Holocene and last glacial distribution. Global and Planetary Change, 40:249-266. doi: 10.1016/j.gloplacha.2003.08.002.

Mollenhauer, G.; Schneider, R.R.; Müller, P.J.; Spieß, V. \& Wefer, G. 2002. Glacial/interglacial variablity in the Benguela upwelling system: Spatial distribution and budgets of organic 
carbon accumulation. Global Biogeochemical Cycles, 16: 81-1-81-15. doi: 10.1029/2001gb001488.

Molnia, B.F. 1974. A Rapid And Accurate Method For The Analysis Of Calcium Carbonate In Small Samples. Journal of Sedimentary Petrology, 44(2):589-590.

Murray, G. \& Blackman, V.H. 1898. On the nature of the Coccospheres and Rhabdospheres. Philosophical Transactions of the Royal Society of London B: Biological Sciences, 190(1): 427-441.

Okada, H. \& Honjo, S.1973. The distribution of oceanic coccolithophores in the Pacific. Deep Sea Research, 20: 355-374.

Paillard, D.; Labeyrie, L. \& Yiou, P. 1996. Macintosh program performs time-series analysis. EOS, Transactions American Geophysical Union, 77: 379.

Pereira, L.S.; Arz, H.W.; Pätzold, J. \& Portilho-Ramos, R.C. 2018. Productivity Evolution in the South Brazilian Bight During the Last 40,000 Years. Paleoceanography and Paleoclimatology, 33:1339-1356. doi: 10.1029/2018PA003406.

Peterson, R.R. \& Stramma, L. 1991. Upper-level circulation in the South Atlantic Ocean. Progrees in Oceanography, 26: 1-73.

Petró, S.M.; Pivel, M.A.G. \& Coimbra, J.C. 2016. Implicações da dissolução de carbonato de cálcio pelágico em reconstruções paleoceanográficas do Quaternário. Quaternary and Environmental Geosciences, 7:14-25.

Pillar, V.D. 1997. Multivariate exploratory analysis and randomization testing with MULTIV. Coenoses, 12: 145-148.

Ravelo, A.C. \& Fairbanks, R.G. 1992. Oxygen Isotopic Composition of multiples species of planktonic foraminifera: records of the modern photic zone temperature gradient. Paleoceanography, 7(6): 815-831.

Reimer, P.J; Bard, E.; Bayliss, A.; Beck, J.W.; Blackwell, P.G.; Ramsey, C.B.; Buck, C.E.; Cheng, H.; Edwards, R.L.; Friedrich, M.; Grootes, P.M.; Guilderson, T.P.; Haflidason, H.; Hajdas, I.; Hatté, C.; Heaton, T.J.; Hogg, A.G.; Hughen, K.A.; Kaiser, K.F.; Kromer, B.; Manning, S.W.; Niu, M.; Reimer, R.W.; Richards, D.A.; Scott, E.M.; Southon, J.R.; Turney, C.S.M. \& Van der Plicht, J. 2013. IntCal13 and MARINE13 radiocarbon age calibration curves 0-50000 years calBP. Radiocarbon, 55: 1869-1887. doi: 10.2458/ azu_js_rc.55.16947.

Schmid, C.; Siedler, G. \& Zenk, W. 2000. Dynamics of intermediate water circulation in the subtropical South Atlantic. Journal of Physical Oceanography, 30: 3191-3211. doi:10.1175/15200485(2000)030<3191:DOIWCI $>2.0 . C O ; 2$.

Silveira, I.C.A.; Scmidt, A.C.K.; Campos, E.J.D.; Godoi, S.S. \& Ikeda, Y. 2000. A Corrente do Brasil ao largo da Costa Leste Brasileira. Revista Brasileira de Oceanografia, 48(2): 171-183.

Stuiver, M. \& Reimer, P.J. 1993. Extended ${ }^{14} \mathrm{C}$ data base and revised Calib $3.0{ }^{14} \mathrm{C}$ age calibration program. Radiocarbon, 35: 215-230.

Van Andel, T.H.; Thiede, J.; Sclater, J.G. \& Hay, W.W. 1977. Depositional history of the South Atlantic Ocean during the last 125 million years. The Journal of geology, 85(6): 651-698.

Young, J.R.; Bown P.R. \& Lees J.A. 2020. Nannotax3 website. International Nannoplankton Association. Disponível em: $<$ http://www.mikrotax.org/Nannotax3>. Acesso em 19 ago. 2020.

Recebido em: 24/07/2020

Aprovado em: 06/10/2020

\section{Como citar:}

Gonçalves, J.F. \& Leonhardt, A. 2021. A Influência dos Processos de Fundo sobre um Registro Fóssil de Cocolitoforídeos na Bacia de Pelotas. Anuário do Instituto de Geociências, 44: 36784. DOI 1982-3908_2021_44_36784 